\title{
CONFORMIDADES E NÃO CONFORMIDADES NO PREPARO E ADMINISTRAÇÃO DE ANTIBACTERIANOS*
}

\author{
Francisco Gilberto Fernandes Pereira ${ }^{1}$, Gabriel Ângelo de Aquino ${ }^{2}$, Geórgia Alcântara Alencar Melo \\ Carolina de Oliveira Praxedes ${ }^{4}$, Joselany Áfio Caetano ${ }^{5}$
}

\begin{abstract}
RESUMO: Objetivou-se avaliar as conformidades e não conformidades no preparo e administração de antibacterianos. Estudo observacional e transversal, realizado de agosto a dezembro de 2014 em unidades de clínica médica de um hospital-escola em Fortaleza-Ceará. A amostra foi composta por 265 doses de antibacterianos, que tiveram suas etapas de preparo e administração observadas com dois checklist nos três turnos de trabalho. Os dados foram analisados por estatística descritiva e analítica, e todos os procedimentos éticos foram seguidos. Destaca-se que a não conformidade mais frequente em ambas as etapas foi o cumprimento dos preceitos da semiotécnica $265(100 \%)$. A organização foi associada com o erro na escolha do medicamento $(p=0,027)$, e o uso da prescrição e confirmação do nome do paciente não tiveram relação com os erros $(p=0,942)$. Assim, conclui-se que modificações comportamentais no processo de trabalho precisam ser implementadas para reduzir as não conformidades e consequentemente os erros de medicação.

DESCRITORES: Antibióticos; Segurança do paciente; Erros de medicação; Enfermagem; Serviços de enfermagem.

\section{COMPLIANCE AND NON-COMPLIANCE IN THE PREPARATION AND ADMINISTRATION OF ANTIBACTERIAL DRUGS}

ABSTRACT: The present study aimed to assess compliance and non-compliance in the preparation and administration of antibacterial drugs. Observational and cross-sectional study conducted from August to December 2014 in medical wards of a teaching hospital in Fortaleza-Ceará. The sample was composed by 265 doses of antibacterial drugs whose preparation and administration stages were observed during the three work shifts, with two checklists. Data were analyzed using descriptive statistics, and all ethical procedures were fulfilled. The most frequent non-compliance observed in both stages was compliance withthe requirements of the several techniques performed by nursing professionals (semiotechnique), 265 (100\%). The hospital organization was associated with error in the choice of the $(\mathrm{p}=0.027)$, and the use of prescription and confirmation of patient's identity were not related to the errors $(p=0.942)$. Thus, it is concluded that behavioral changes in the work process should be implemented to reduce non-compliance and, consequently, medication errors.

DESCRIPTORS: Antibiotics; Patient safety; Medication errors; Nursing; Nursing services.
\end{abstract}

\section{CUMPLIMIENTO Y INCUMPLIMIENTO EN EL PREPARO Y ADMINISTRACCIÓN DE ANTIBACTERIANOS: IMPLICACIONES PARA LA SEGURIDAD DEL PACIENTE}

RESUMEN: El presente estudio tuvo como objetivo evaluar el cumplimiento y el incumplimiento en la preparación y administración de fármacos antibacterianos. Estudio observacional y transversal realizado entre agosto y diciembre de 2014 en las alas médicas de un hospital universitario en Fortaleza-Ceará. La muestra fue compuesta por 265 dosis de fármacos antibacterianos cuya etapas del preparación y administración fueron observados durante los tres turnos de trabajo, con dos listas de comprobación. Los datos fueron analizados utilizando estadística descriptiva, y se cumplieron todos los procedimientos éticos. El incumplimiento más frecuentes observados en las dos etapas era el cumplimiento de los requisitos de las varias técnicas realizadas por los profesionales de enfermería (semiología), $265(100 \%)$. La organización del hospital se asoció con error en la elección dela ( $p=0,027)$, y el uso de la prescripción y de la confirmación de la identidaddel paciente no estaban relacionados con los errores $(p=0,942)$. Por lo tanto, se concluye que los cambios de comportamiento en el proceso de trabajo se deben implementar para reducir el incumplimiento y, en consecuencia, los errores de medicación.

DESCRIPTORES: Antibióticos; Seguridad del paciente; Errores de medicación; Enfermería; Servicios de enfermería.

*Artigo extraído da dissertação intitulada: "Erros de medicação antibacteriana e a interface com a segurança do paciente". Universidade Federal do Ceará, 2015.

${ }^{1}$ Enfermeiro. Mestre em Enfermagem. Docente de Enfermagem da Universidade Federal do Piauí. Picos, PI, Brasil.

${ }^{2}$ Discente de Enfermagem. Universidade Federal do Ceará. Fortaleza, CE, Brasil.

${ }^{3}$ Enfermeira. Mestranda em Enfermagem. Universidade Federal do Ceará. Fortaleza, CE, Brasil.

${ }^{4}$ Enfermeira. Centro Universitário Estácio do Ceará. Hospital Antônio Prudente. Fortaleza, CE, Brasil.

${ }^{5}$ Enfermeira. Doutora em Enfermagem. Docente do Programa de Pós-graduação em Enfermagem da Universidade Federal do Ceará. Fortaleza, CE, Brasil.

Autor Correspondente:

Francisco Gilberto Fernandes Pereira

Recebido: $26 / 02 / 2016$

Universidade Federal do Piauí

R. Professor Vicente Silveira, 100 - 60410-322 - Fortaleza, CE, Brasil

E-mail: gilberto.fp@hotmail.com 


\section{INTRODUÇÃO}

As situações de não conformidades no preparo e administração de medicamentos constituem um problema de saúde pública devido a sua grande ocorrência, principalmente quando se refere aos antibacterianos, pois o manuseio inadequado dessa classe farmacológica acarreta gastos adicionais para o sistema de saúde, por estar diretamente relacionado à resistência bacteriana. Assim, torna-se necessário o real conhecimento da ocorrência de conformidades e não conformidades no preparo e administração de antibacterianos.

A equipe de enfermagem tem como uma responsabilidade assegurar vigilância rigorosa na administração de antibacterianos, assim como o controle dos horários, diluições e intervalos entre as doses, evitando dessa forma a seleção de microrganismos resistentes devido a níveis plasmáticos inapropriados. Tal condição delibera que essa prática seja exercida de forma correta e segura aos pacientes e que, portanto, os erros sejam minimizados e prevenidos.

No cenário mundial, observa-se que erro na administração das medicações na África Oriental é comum, com uma taxa que varia de $9,4 \%$ a $80 \%{ }^{(1)}$. Ainda na África, dentro do cenário de terapia intensiva adulta e pediátrica, a prevalência de erro na administração das medicações mostrou $51,8 \%$ e $90,8 \%$, respectivamente ${ }^{(2)}$. No Reino Unido, $26 \%$ do erro na administração das medicações eram potencialmente graves, com eventos fatais que levaram à pneumonia aspirativa e hemorragia intracraniana ${ }^{(3)}$.

No cenário brasileiro, um estudo multicêntrico realizado em cinco capitais demonstrou que em relação ao sistema de medicação, 39\% dos erros ocorrem durante a prescrição, $12 \%$ na transcrição, $11 \%$ na dispensação e 38\% durante a administração. Mostrou também que enfermeiros e farmacêuticos interceptaram $86 \%$ dos erros de medicação relacionados à prescrição, transcrição e dispensação, enquanto apenas $2 \%$ são interceptados pelos pacientes. Quanto à classe farmacológica mais envolvida nos erros, destacaram-se os antimicrobianos, com uma variação de 8,4\% a 18,5\%, destacando-se os erros de horário $(87,7 \%)$ e de dose $(6,9 \%)$ como os mais constantes ${ }^{(4)}$.

Erro na administração das medicações tem um impacto significativo sobre os pacientes em termos de morbidade, mortalidade, evento adverso de drogas e aumento de tempo de permanência hospitalar, além de aumentar os custos para os sistemas de saúde ${ }^{(5)}$.

Os fatores que mais contribuem para erros na administração de medicação incluem aspectos organizacionais, tais como as interrupções, o armazenamento incorreto, o tempo do preparo, a prescrição médica ilegível, a má comunicação e a não verificação do nome do paciente antes da administração ${ }^{(6-7)}$.

Justifica-se a relevância de uma pesquisa que relacione os erros de medicação com aspectos organizacionais do processo de trabalho, compreendendo-se que é importante conhecer o cenário para se tentar evitar falhas que comprometam a segurança do paciente e a eficácia do medicamento.

Nesse contexto, o presente estudo tem o objetivo de avaliar as conformidades e não conformidades no preparo e administração de antibacterianos.

\section{MÉTODO}

Trata-se de um estudo observacional, transversal, realizado entre os meses de agosto a dezembro de 2014 nos setores de clínica médica em um hospital de ensino pertencente à Rede Sentinela da Agência Nacional de Vigilância Sanitária (ANVISA), localizado em Fortaleza-Ceará. Os setores estão divididos em dois postos de enfermagem, e de modo geral, são responsáveis por uma ocupação de 114 leitos distribuídos no atendimento às seguintes especialidades: dermatologia, cardiologia, clínica médica, endocrinologia, gastroenterologia, pneumologia, neurologia, nefrologia, hematologia e reumatologia.

A coleta de dados se operacionalizou em dias e turnos sorteados pelos pesquisadores de modo a contemplar os períodos matutino, vespertino e noturno. Para que se pudesse definir a quantidade de doses a serem observadas, ou seja, a amostra, solicitou-se ao serviço de farmácia hospitalar da 
instituição a informação sobre a quantidade de doses dos antibacterianos, seccionada por turnos, dispensadas para as clínicas A e B em um período de trinta dias anteriores ao início da coleta de dados. Foram incluídos apenas os antibacterianos, devido à estreita relação que se conhece entre variações de condições ambientais e a alteração da sua estabilidade química ${ }^{(8)}$.

Assim, o grupo amostral da pesquisa foi formado por $40 \%$ das doses informadas pelo serviço de farmácia. Este percentual amostral foi calculado com base na média de erros de preparo e administração de medicação antimicrobiana presentes em outro estudo brasileiro ${ }^{(9)}$.

Foram realizadas na Clínica Médica B 157 observações: 67 no período da manhã, 30 à tarde e 60 durante a noite. Já na Clínica Médica A, foram observadas 108 doses: 44 pela manhã, 22 no período vespertino e 42 no plantão noturno.

Para atingir o objetivo proposto, as observações estruturadas foram realizadas tendo início no momento do preparo e finalizadas na administração completa do medicamento. Nesse período, os observadores utilizaram dois instrumentos do tipo checklist proposto por Cassiani ${ }^{(10)}$, contendo as seguintes variáveis: preparo (organização, interrupções, semiotécnica, existência de protocolos, rotulagem do medicamento e utilização da prescrição); e, durante a administração (organização, interrupções, semiotécnica, utilização da prescrição, identificação do paciente, controle da infusão, checagem da administração e monitoramento).

Considerou-se que a conformidade das atividades ou processos consiste em atender a um determinado padrão, capaz de definir a qualidade almejada de maneira a não comprometer a coerência e o preconizado pelo serviço, constatada no decorrer da avaliação(11). Neste caso, a conformidade foi considerada quando houve coerência total entre a situação observada e os critérios estabelecidos no instrumento de coleta de dados. Já não-conformidade foi pontuada quando houve descumprimento destes critérios.

Com o intuito de evitar viés na coleta de dados, a equipe de pesquisadores passou por capacitação diária, orientado pelo pesquisador responsável, num período de 30 dias; houve padronização dos conceitos observados, embasados em literatura científica e treinamento prévio com os instrumentos de coleta de dados. E, para evitar o efeito Hawthorne, que é comum em estudos observacionais, as 20 observações realizadas no mês de agosto não foram consideradas para avaliação.

Os dados obtidos foram agrupados no banco de dados com auxílio do Statistical Software for the Social Science (SPSS) 19.0 para julgamento estatístico e os testes de Kolmogorov-Smirnove MannWhitney foram realizados para identificar as associações entre as variáveis levantadas e os erros de medicação.

Vale ressaltar que o estudo se fundamentou na classificação de erro utilizada pelo National Coordinating Council for Medication Error Reporting and Prevention (NCC-MERP) ${ }^{(12)}$, que caracteriza o erro de acordo com seu potencial de dano ao paciente em nove categorias de erro em função da gravidade, considerando se houve ou não danos ao paciente, qual a duração e a extensão deste e se foi necessária alguma intervenção. A categoria A (quase erro) são as circunstâncias ou acontecimentos susceptíveis de originarem um erro; categoria B houve o erro, porém não atingiu o paciente; categoria $\mathrm{C}$ o erro atinge o paciente, no entanto não causa danos; categoria $\mathrm{D}$ o erro atinge o paciente e requer monitoramento para confirmar se houve dano ao paciente e/ou requer intervenção para impedir o mesmo. As categorias E, F, G e H são classificados como erro com dano ao paciente e categoria I como erro seguido de morte. $E$, na ocorrência do erro, optou-se por intervir sempre que houvesse algum erro, orientando-se o funcionário quanto à forma correta e segura de administrar o medicamento.

O projeto foi enviado ao Comitê de Ética em Pesquisa da Universidade Federal do Ceará e obteve como aprovação o $\mathrm{n}^{\circ} 660.897$. Foram respeitados todos os princípios éticos de estudos nacionais e internacionais.

\section{RESULTADOS}

Considerando as variáveis investigadas neste estudo, vale ressaltar que a ação de não-conformidade mais frequente nas etapas de preparo e administração de antibacterianos nos dois setores foi o 
descumprimento dos preceitos da semiotécnica 265 (100\%), conforme apresentado na Tabela 1, evidenciado por ausência de desinfecção das ampolas do medicamento, não utilização de luvas durante o procedimento, respingos de antibacteriano dispersados no ar, contaminação do êmbolo das seringas, e preparo com antecedência superior a 30 minutos do momento da administração. Estes subitens não foram quantificados durante a coleta dos dados, pois importava apenas identificar se havia ou não erro de técnica.

Ao analisar as variáveis específicas de cada fase, houve maior frequência de ações de nãoconformidade na rotulagem do medicamento 265 (100\%) para o preparo, e uso da prescrição para confirmar os dados do medicamento e do paciente 243 (91,7\%) no momento da administração do antibacteriano (Tabela 1). Contraditoriamente, durante o processo de preparo, foi constatado maior grau de conformidade no item que avaliou o uso da prescrição para confirmação do nome do paciente, $222(83,8 \%)$ observações.

Na etapa de administração dos medicamentos foram observadas 147 (55,5\%) conformidades no que concerne ao item interrupção; entretanto no momento do preparo teve-se 145 (54,7\%) nãoconformidades e estão relacionados à utilização de aparelhos de som no ambiente, fones de ouvido conectados a aparelhos multimídia, e maior número de profissionais dividindo o mesmo espaço físico no setor.

Os itens de checagem do medicamento administrado, monitoramento e controle do tempo de infusão foram mais incidentes para não-conformidades, com $172(64,9 \%), 166(62,7 \%)$ e 159 (60\%), respectivamente.

Quanto ao aspecto da organização, foi observada inadequação em 187 (70,6\%) e 138 (52,1\%) doses nas fases de preparo e administração, respectivamente. No período matutino se constatou maior frequência desta característica.

Ainda sobre a organização no ambiente de preparo, observou-se que os auxiliares e técnicos ficam com uma média de 5-6 pacientes por período, e quando assumem o plantão, colocam as prescrições dos pacientes numa prancheta separada do prontuário, e a seguir realizam a separação dos medicamentos e os identificam com etiquetas com número do leito e horário da administração do medicamento.

Tabela 1 - Conformidades e não-conformidades nas etapas de preparo e administração de antibacterianos. Fortaleza, CE, Brasil, 2014

\begin{tabular}{|c|c|c|c|}
\hline Característica & $\begin{array}{c}\text { Conformidade } \\
\text { n(\%) }\end{array}$ & $\begin{array}{c}\text { Não-conformidade } \\
\mathbf{n}(\%)\end{array}$ & $\begin{array}{l}\text { Total } \\
\text { n(\%) }\end{array}$ \\
\hline \multicolumn{4}{|l|}{ Preparo } \\
\hline Organização & $78(29,4)$ & $187(70,6)$ & $265(100)$ \\
\hline Interrupções & $120(45,3)$ & $145(54,7)$ & $265(100)$ \\
\hline Uso da prescrição & $222(83,8)$ & $43(16,2)$ & $265(100)$ \\
\hline Semiotécnica & - & $265(100)$ & $265(100)$ \\
\hline Rotulagem correta & - & $265(100)$ & $265(100)$ \\
\hline \multicolumn{4}{|l|}{ Administração } \\
\hline Organização & $127(47,9)$ & $138(52,1)$ & $265(100)$ \\
\hline Interrupções & $147(55,5)$ & $118(45,5)$ & $265(100)$ \\
\hline Uso da prescrição & $22(8,3)$ & $243(91,7)$ & $265(100)$ \\
\hline Confirmação do nome do paciente & $55(20,7)$ & $210(79,3)$ & $265(100)$ \\
\hline Semiotécnica & - & $265(100)$ & $265(100)$ \\
\hline Controle do tempo de infusão & $106(40)$ & $159(60)$ & $265(100)$ \\
\hline Checagem do medicamento administrado & $93(35,1)$ & $172(64,9)$ & $265(100)$ \\
\hline Monitoramento & $99(37,3)$ & $166(62,7)$ & $265(100)$ \\
\hline
\end{tabular}

Fonte: Dados do pesquisador 
Ao avaliar comportamento específicos da etapa de administração, nota-se uma prevalência de não-conformidades quanto ao comportamento de utilização da prescrição, confirmação do nome do paciente e monitoramento. E, na clínica B os maiores índices de conformidades foram controle de infusão e checagem imediata.

Fez-se, ainda, a associação da organização no preparo e a escolha errada do medicamento nesta etapa. Foi realizado o teste de Fisher, pois as hipóteses do teste qui-quadrado não foram satisfeitas. Na Tabela 2 nota-se que a maior concentração dos dados está na relação das categorias Não-Não quando se somam as duas clínicas. Por meio do Teste de Fisher calculou-se o p-valor que foi de 0,027, demonstrando desta forma que existe associação entre as duas variáveis.

Na Tabela 3 encontra-se a relação entre o uso da prescrição e a confirmação do nome do paciente na administração do medicamento, o p-valor foi de 0,942 , evidenciando que não houve associação entre essas duas variáveis. Vale ressaltar que os pacientes são identificados apenas pelo número do leito. Por vezes, na placa de identificação localizada acima do leito, havia também o nome do paciente e data de admissão. Assim, na Clínica A, apenas oito trabalhadores de enfermagem usaram a prescrição e confirmaram o nome, já na Clínica B, durante as observações $100 \%$ não confirmou o nome do paciente por meio da prescrição e nem a utilizou no momento da administração do medicamento. Vale ressaltar que no geral todos os erros encontrados foram do tipo B, ou seja, um erro ocorreu, mas não chegou a atingir o paciente.

Tabela 2 - Associação entre as variáveis organização e escolha errada do medicamento no preparo de antibacterianos nas Clínicas Médicas A e B. Fortaleza, CE, Brasil, 2014

\begin{tabular}{|c|c|c|c|c|c|}
\hline & \multirow[t]{2}{*}{$\begin{array}{c}\text { Organização } \\
\text { (preparo) }\end{array}$} & \multicolumn{2}{|c|}{$\begin{array}{l}\text { Escolha errada do } \\
\text { medicamento (preparo) }\end{array}$} & \multirow[t]{2}{*}{ Total } & \multirow[t]{2}{*}{ p-valor* } \\
\hline & & Sim & Não & & \\
\hline \multirow[t]{3}{*}{ CLÍNICA MÉDICA A } & Sim & 1 & 53 & 54 & \multirow[t]{3}{*}{0,113} \\
\hline & Não & 6 & 48 & 54 & \\
\hline & Total & 7 & 101 & 108 & \\
\hline \multirow[t]{3}{*}{ CLÍNICA MÉDICA B } & Sim & 0 & 24 & 24 & \multirow[t]{3}{*}{0,027} \\
\hline & Não & 22 & 111 & 133 & \\
\hline & Total & 22 & 135 & 157 & \\
\hline
\end{tabular}

Fonte: Dados do pesquisador

*Teste exato de Fisher

Tabela 3 - Associação entre usou a prescrição e confirmou o nome do paciente na administração do medicamento na Clínica A. Fortaleza, CE, Brasil, 2014

\begin{tabular}{lccccc} 
Usou a prescrição (Administração) & \multicolumn{2}{c}{$\begin{array}{c}\text { Confirmou o nome do } \\
\text { paciente (administração) }\end{array}$} & \multirow{2}{*}{ Total } & p-valor* \\
\cline { 2 - 4 } & Sim & Não & & \\
\hline Sim & 8 & 14 & 22 & 0,942 \\
\hline Não & 32 & 54 & 86 \\
\hline Total & 40 & 68 & 108 &
\end{tabular}

Fonte: Dados do pesquisador

* Teste exato de Fisher

\section{- DISCUSSÃO}

Neste estudo encontrou-se que a realização das etapas de preparo e administração dos antibacterianos é realizada eminentemente por técnicos e auxiliares de enfermagem, o que não diverge das realidades descritas em outra pesquisa conduzida em instituições brasileiras ${ }^{(13)}$. 
O período matutino foi onde se constatou maior frequência desta característica, o que pode estar relacionado à maior circulação de profissionais de saúde no setor e também maior fluxo de preparo de medicamento, como também ser o horário dos ajustes da prescrição de antibacterianos pela equipe médica nestas unidades. Em até $87 \%$ dos casos de erros de medicação, a responsabilidade recai sobre os fatores humanos e insuficiências organizacionais ${ }^{(14)}$. A experiência cotidiana sugere que a elevada carga de trabalho, o número inadequado de enfermeiros experientes, a supervisão inadequada e a omissão de feedback dos enfermeiros sobre os erros, bem como distrações, má comunicação, pressa e fadiga são fatores contribuintes para os erros.

Há condições que podem influenciar negativamente na dinâmica de trabalho dos profissionais, gerar distrações e facilitar a ocorrência de lapsos, das quais se destacam: a composição do perfil sócio ocupacional do profissional; e as interferências ambientais a que estão submetidos, como por exemplo, a superlotação nas unidades e o inadequado dimensionamento arquitetônico dos espaços.

Neste estudo, o erro predominante foi de administração relacionado basicamente à execução dos preceitos da semiotécnica, em 265 (100\%) observações. Este dado corrobora com estudo realizado em dois hospitais no Vietnã, que observou um total de 5271 administrações de medicamentos. A técnica errada de administração estava presente em (23,5\%) das observações, seguido de erro na técnica da preparação, a omissão, e dose errada dos medicamentos $(15,7 \%, 2,3 \% \text { e 1,8\%, respectivamente })^{(15)}$.

De acordo com uma revisão sobre erros de medicação por via intravenosa ${ }^{(16)}$, observou-se com frequência erros na técnica de administração, técnica de preparação, omissão e erros de dose. Cerca de metade dos erros foi técnica de administração errada e a maioria destes era erros na velocidade da infusão envolvendo medicamentos em bolus, que deveriam ter sido administrados em três a cinco minutos.

No tocante às variáveis comportamentais, fatores que geram interrupções na execução da tarefa de preparar os medicamentos foram percebidos com alta frequência durante a realização da pesquisa, sendo 145 doses com interrupção durante o preparo e 118 na administração. Estas foram produzidas por diferentes fontes, tais como: frequente tráfego de pessoas, conversas paralelas, utilização de aparelho de som do tipo micro system e uso de fones de ouvido conectados a smartphones. Este cenário é incoerente ao recomendado, que deveria ser um ambiente seja reservado, com fluxo restrito de pessoas, tranquilo e sem fonte de interrupção ou distração, como televisão e/ou rádio ${ }^{(17)}$.

Em um estudo realizado em dois hospitais na Austrália, verificou-se que o aumento do número de interrupções foi ligado ao aumento das taxas de erro ${ }^{(18)}$. Entre os fatores que provocam distrações durante as tarefas de trabalho dos profissionais de enfermagem estão: ausência de planejamento formal da assistência de enfermagem, telefone celular e/ou institucional tocando, sobreposição de diferentes tarefas, mudanças não padronizadas de prescrição médica e interrupções geradas por outros profissionais ${ }^{(19)}$.

Algumas estratégias têm sido empregadas para reduzir potenciais interrupções durante o preparo e administração de medicamentos, como por exemplo, a utilização de coletes com cores de destaque e frases de efeito do tipo "não perturbe" para o profissional responsável por esta função. Mas seus idealizadores apontam que, embora esta alternativa tenha apresentado redução significativa no número de erros de medicamentos relacionados às interrupções, os pacientes têm demonstrado certo grau de resignação e desumanização na assistência, pois acreditam que as mensagens sejam direcionadas a eles ${ }^{(20)}$.

No que concerne à organização, estudo(21) explica que a estrutura física dos diversos ambientes que compõem o hospital deve se concentrar em atender três pré-requisitos: funcionais, técnicos e psicossociais. Assim, no contexto da funcionalidade, prover e manter o ambiente organizado é um fator que certamente facilita os processos de trabalho, agiliza a realização de tarefas e, principalmente, coopera com a prestação de cuidados mais seguros.

Detectou-se que os fatores ergonômicos da sala de preparo de medicamentos em um hospital escola de Florianópolis ${ }^{(22)}$ foram similares aos descritos nos resultados desta pesquisa, como por exemplo: gavetas faltando divisórias, limitação do espaço para preparo do medicamento, poluição visual e sonora, e fluxo intenso entre os profissionais do registro onde a prescrição medicamentosa é realizada. 
Por meio de uma revisão integrativa em que foram avaliadas 25 publicações internacionais ${ }^{(23)}$, concluiu-se que a organização do ambiente é um fator basilar para a prevenção de quaisquer eventos adversos relacionados à prestação de cuidados em saúde, e realçaram que na maioria dos estudos revisados houve uma multiplicidade de tarefas neste ambiente, o que inviabiliza a correta estruturação do sistema de medicação.

Estudo confirma que os métodos utilizados durante o preparo de medicamentos são falhos na maioria dos sistemas de medicação, e a organização ao desempenhar esta tarefa é afetada negativamente pela escassez ou má utilização de materiais, disposição não funcional de mobiliários (bancadas pequenas, gavetas sem identificação) e ambiente multitarefas ${ }^{(24)}$.

Outro aspecto importante diz respeito à conferência do nome do paciente, que compreende uma das nove certezas no que se refere a limitar os erros durante a administração dos medicamentos. Geralmente a omissão da etapa de identificação do nome do paciente é justificada pela equipe de enfermagem em face ao longo tempo de internação que cria certo vínculo entre a equipe e o paciente, tornando assim desnecessária sua realização. Isto está fortemente associado à forma como a enfermagem se habituou a organizar o processo de trabalho, pois, embora não houvesse o erro, isto não deixa de ser um método falho no sistema. Neste aspecto, houve o mesmo padrão de desempenho por parte da equipe, já que não se observou diferenças significativas no processo de administração dos antibacterianos nas unidades, evidenciando que quando os erros acontecem, tem origem sistêmica, podendo ser resultado do processo de trabalho da instituição.

Conforme recomendações do Ministério da Saúde ${ }^{(17)}$, a confirmação do nome do paciente deve ser realizada por meio de dois identificadores: a identificação nominal no prontuário e a data de nascimento ou número do prontuário; devendo ocorrer sempre antes de qualquer cuidado, inclusive na administração de medicamentos. A realidade observada nesta pesquisa de não checar o nome do paciente por já estar internado na unidade há muitos dias, deve ser abolida quando a intenção for oferecer assistência segura.

Este aspecto pressupõe que o longo tempo de permanência do paciente no setor e a rotina dos antibacterianos utilizados favorece uma relação mais próxima deste com a equipe, permitindo conhecêlos fisionomicamente, dispensando-a desta forma a usar a prescrição para checar o nome do paciente ou seu medicamento na ocasião da administração medicamentosa. Percebe-se que denominar o paciente pelo leito ainda é uma forma habitual de comunicação entre as equipes de saúde.

Porém, o risco de um erro de medicação ou evento adverso é proporcional ao tempo de internação, ou seja, quanto mais tempo o indivíduo fica hospitalizado, maiores são as chances de estes incidentes acontecerem. Para os antibióticos essa probabilidade é ainda maior ${ }^{(25-26)}$.

No caso específico dos antibacterianos, essa checagem é importantíssima, pois a indicação do medicamento é realizada de acordo com critérios clínico-laboratoriais do perfil de resistência e sensibilidade das bactérias, e, caso o antibiótico seja administrado ao paciente errado, pode-se gerar um quadro de resistência microbiana que culminará em curto prazo com o agravamento do quadro clínico do indivíduo, levando inclusive a septicemias.

\section{- CONCLUSÃO}

A pesquisa indica que rotina de trabalho e fatores organizacionais contribuem para os erros na administração de medicamentos. Assim, neste estudo, os erros de medicação na administração nas fases de preparo e administração foram da categoria $B$, sem dano ao paciente. Todas as observações foram acompanhadas de, pelo menos, um tipo de erro. A não-conformidade na semiotécnica foi o tipo mais dominante, seguida da rotulagem.

Fatores organizacionais tais comolimpeza, organização espacial de forma ter melhor aproveitamento do espaço, redução de pessoas circulante na sala, redução da poluição visual e sonora, notificação de sistemas de relatórios de erro e verificações de rotina poderiam eventualmente ajudar no tratamento do problema dos erros de medicação.

Assim, novos estudos devem ser realizados em outros centros, para que se comparem os seus 
resultados, e também estabelecer estratégias para garantir a segurança do paciente, inclusive propor estratégias e avaliá-las quanto ao êxito na redução do problema.

Como limitação destaca-se que a estrutura física da instituição do estudo favorece ao erro no preparo de medicação, pois o local de preparo de medicamento apresenta variáveis ambientais que geram desconforto para os profissionais de enfermagem, além de ser um local onde grande variedade de atividades são realizadas.

\section{REFERÊNCIAS}

1. Alsulami Z, Conroy S, Choonara I. Medication errors in the Middle East countries: A systematic review of the literature. Eur J Clin Pharmacol. [Internet] 2013; 69(4) [acesso em 12 nov 2015]. Disponível:

https://dx.doi.org/10.1007/s00228-012-1435-y.

2. Agalu A, Ayele Y, Bedada W, Woldie M. Medication administration errors in an intensive care unit in Ethiopia. Int Arch Med. [Internet] 2012; 5(1) [acesso em 10 out 2015]. Disponível: http://dx.doi.org/10.1186/1755-7682-5-15.

3. Adams M, Koch R. Pharmacology: Connections to Nursing Practice. New Jersey: Pearson; 2010.

4. Marques TC, Reis AMM, Silva AEBC, Gimenes FRE, Opitz SP, Teixeira TCA, et al. Erros de administração de antimicrobianos identificados em um estudo multicêntrico brasileiro. Rev. Bras. Cienc. Farm. [Internet] 2008; 44(22) [acesso em 20 fev 2016]. Disponível: http://dx.doi.org/10.1590/S1516-93322008000200016.

5. Popescu A, Currey J, Botti M. Multifactorial influences on and deviations from medication administration safety and quality in the acute medical/ surgical context. Worldviews Evid Based Nurse. [Internet] 2011; 8(1) [acesso em 20 jan 2016]. Disponível: https://dx.doi.org/10.1111/j.1741-6787.2010.00212.x.

6. Feleke SA, Mulatu MA, Yesman YS. Medication administration error: magnitude and associated factors among nurses in Ethiopia. BMC Nursing. [Internet] 2015; 14:53 [acesso em 20 jul 2015]. Disponível:

https://dx.doi.org/10.1186/s12912-015-0099-1.

7. Kane-Gill S, Kowiatek JG, Weber RJ. A comparison of voluntarily reported medication errors in intensive care and general care units. Qual Saf Health Care. [Internet] 2010; 19: 55 [acesso em 20 jul 2015]. Disponível:

https://dx.doi.org/10.1136/qshc.2008.027961.

8. Crepaldi RMC, Monteiro C, Peterlini MAS, Pedreira MLG. Hydrogen-ion potential of antibiotics according to the environment factors temperature and luminosity. Rev. Latino-Am. Enfermagem. [Internet] 2010; 18(2) [acesso em 20 jul 2015]. Disponível: http://dx.doi.org/10.1590/S0104-11692010000200020.

9. Silva AEBC, Cassiani SHB. Prospective risk of the anti-infective medication administration process. Rev. LatinoAm Enfermagem. [Internet] 2013; 21(n. spe) [acesso em 8 out 2015]. Disponível:

http://dx.doi.org/10.1590/S0104-11692013000700029.

10. Cassini SHB. Identificação e análise dos erros de medicação em hospitais brasileiros. 2006 [tese]. Ribeirão Preto (SP): Universidade de São Paulo; 2006.

11. Organização Nacional de Acreditação (ONA). Manual Brasileiro de Acreditação. $6^{a}$ ed. Brasília: ONA; 2010.

12. National Coordinating Council for Medication Error Reporting and Prevention (NCCMERP). Taxonomy of medication errors. Estados Unidos. [Internet] 2001 [acesso em 20 fev 2016]. Disponível:

http://www.nccmerp.org/taxonomy-medication-errors-now-available.

13. Carneiro FS, Bezerra ALQ, Silva AEBC, de Souza LP, Paranaguá TTB, Branquinho NCSS. Eventos adversos na clínica cirúrgica de um hospital universitário: instrumento de avaliação da qualidade. Rev. enferm. UERJ. [Internet] 2011; 19(2) [acesso em 20 jul 2014]. Disponível: http://www.facenf.uerj.br/v19n2/v19n2a06.pdf.

14. Harolds JA, Harolds LB. Quality and safety in health care, Part VIII: Introduction to medication safety. Clin Nucl Med. [Internet] 2016; 41(3) [acesso em 20 fev 2016]. Disponível: https://dx.doi.org/10.1097/RLU.0000000000001097.

15. Nguyen HT, Nguyen TD, van den Heuvel ER, Haaijer-Ruskamp FM, Taxis K. Medication errors in Vietnamese hospitals: prevalence, potential outcome and associated factors. PLoS ONE. [Internet] 2015 [acesso em 20 jan 
2016]. Disponível: http://dx.doi.org/10.1371/journal.pone.0138284.

16. McDowell SE, Mt-Isa S, Ashby D, Ferner RE. Where errors occur in the preparation and administration of intravenous medicines: A systematic review and Bayesian analysis. Qual Saf Health Care. [Internet] 2010; (19) [acesso em 20 jan 2016]. Disponível: https://dx.doi.org/10.1136/qshc.2008.029785.

17. Ministério da Saúde (BR). Portaria n. 529, de $1^{\circ}$ de abril de 2013. Institui o Programa Nacional de Segurança do Paciente (PNSP). [Internet] Brasília: Ministério da Saúde; 2013 [acesso em 20 jul 2014]. Disponível:

http://bvsms.saude.gov.br/bvs/saudelegis/gm/2013/prt0529_01_04_2013.html.

18. Westbrook JI, Woods A, Rob MI, Dunsmuir WTM, Day RO. Association of interruptions with an increased risk and severity of medication administration errors. Arch Intern Med. [Internet] 2010; 170(8) [acesso em 20 jul 2014] Disponível: https://dx.doi.org/10.1001/archinternmed.2010.65.

19. Lemos NRF, da Silva VR, Martinez MR. Fatores que predispõem à distração da equipe de enfermagem durante o preparo e administração de medicamentos. Reme, Rev. Min. Enferm. [Internet] 2012; 16(2) [acesso em 20 jul 2014]. Disponível: http://www.reme.org.br/artigo/detalhes/520.

20. Palese A, Ferro M, Pascolo M, Dante A, Vecchiato S. "I am administration medication-please do not interrupt me": red tabards preventing interruptions as perceived by surgical patients. Journal Patient Safety. [Internet] 2015; 7(4) [acesso em 20 jul 2015] Disponível: https://dx.doi.org/10.1097/PTS.0000000000000209.

21. Lima CD, Lopes MA, Gonçalves VMS. O enfermeiro no planejamento do espaçço físico hospitalar. Revista Enfermagem Integrada. [Internet] 2010; 3(2) [acesso em 20 jul 2015]. Disponível: http://www.unilestemg.br/ enfermagemintegrada/artigo/V3_2/02-enfermeiro-no-planejamento-fisico-hospitalar.pdf.

22. Pichler RF, Garcia LJ, Seitz EM, Merino GSAD, Gontijo LA, Merino EAD. Erro de medicação: análise ergonômica de utensílios da sala de medicação em ambiente hospitalar. Cad. Saúde Colet. [Internet] 2014; 22(4) [acesso em 03 de maio de 2015] Disponível: http://dx.doi.org/10.1590/1414-462X201400040004.

23. Santana JCB, de Sousa MA, Soares HC, Avelino KSA. Fatores que influenciam e minimizam os erros na administração de medicamentos pela equipe de enfermagem. Enferm. rev. [Internet] 2012; 15(1) [acesso em 20 jul 2015]. Disponível: http://periodicos.pucminas.br/index.php/enfermagemrevista/article/view/3300/3657.

24. Liu W, Manias E, Gerdtz M. The effects of physical environments in medical wards on medication communication processes affecting patient safety. Health Place. [Internet] 2014; (26) [acesso em 20 jul 2015] Disponível: http://dx.doi.org/10.1016/j.healthplace.2013.12.017.

25. Duarte SCM, Stipp MAC, Silva MM, de Oliveira FT. Adverse events and safety in nursing care. Rev. bras. enferm. [Internet] 2015; 68(1) [acesso em 20 abr 2015] Disponível: http://dx.doi.org/10.1590/0034-7167.2015680120p.

26. Silva MDG, Rosa MB, Franklin BD, Reis AMM, Anchieta LM, Mota JAC. Concomitant prescribing and dispensing errors at a Brazilian hospital: a descriptive study. Clinics. [Internet] 2011; 66(10) [acesso em 20 abril 2015] Disponível: http://dx.doi.org/10.1590/S1807-59322011001000005. 